\title{
An abnormal displacement change during holding period in nanoindentation tests on zirconia dental ceramic
}

\author{
Lixian $\mathrm{ZHANG}^{a}$, Longquan $\mathrm{SHAO}^{b}$, Lei $\mathrm{LI}^{c}$, Danyu JIANG ${ }^{c, *}$ \\ ${ }^{a}$ Department of Prosthodontics, the First Hospital of Yunnan Province, Kunming 650000, China \\ ${ }^{b}$ Department of Stomatology, Nanfang Hospital, Southern Medical University, Guangzhou 510515, China \\ ${ }^{c}$ Analysis and Testing Center for Inorganic Materials, the State Key Laboratory of High Performance Ceramics \\ and Superfine Microstructure, Shanghai Institute of Ceramics, Shanghai 200050, China
}

Received: February 28, 2016; Accepted: March 07, 2016

(C) The Author(s) 2016. This article is published with open access at Springerlink.com

\begin{abstract}
An abnormal displacement change observed during the holding period in nanoindentation tests on a zirconia dental ceramic was reported in this paper. It was found that, at the initial stage of the holding period, the measured displacement versus time curves were similar in shape with the typical indentation creep curve reported in previous studies. As the holding lasted for long time, however, an evident reduction in displacement was observed for tests with high loading rate, implying that another unknown process, which might result in a decrease in displacement, would co-exist with creep during holding period. Elastic recovery was suggested to be one of the possible sources for such a displacement reduction. An empirical method was also proposed to eliminate the effect of this displacement reduction on the determination of hardness and Young's modulus.
\end{abstract}

Keywords: zirconia; nanoindentation; displacement; elastic recovery

\section{Introduction}

In a nanoindentation test, a load-displacement curve is recorded during the indenter is pushed into the sample surface (loading segment), held at peak loading for a certain duration (holding segment), and then withdrawn from the sample surface (unloading segment). In most of the previous studies using nanoindentation test to characterize mechanical properties of materials, attentions are only paid on the unloading segment because the hardness and the Young's modulus are usually extracted from the analysis of the unloading behavior based on the well-known Oliver-Pharr (OP) method [1]. The loading segment is occasionally observed mainly aiming at providing experimental

\footnotetext{
* Corresponding author.

E-mail: dyjiang@mail.sic.ac.cn
}

supports to the theoretical analyses of the load-displacement relationship [2,3]. Some studies are also concentrated on the holding segment, but the purpose of such studies is to observe the nanoindentation creep behavior [4,5]. Little effort has been paid on effect of the deformation occurring during holding period on the determination of hardness and Young's modulus.

Evaluation of the resistance to contact damage is a critical issue for the clinical applications of zirconia dental ceramics [6]. As an important parameter for evaluating the material's resistance to contact damage, hardness characteristics of zirconia dental ceramics have been frequently examined with nanoindentation tests in many studies during the past years [6-9].

This paper will report an abnormal phenomenon observed during holding period in nanoindentation tests conducted on an yttria-stabilized zirconia tetragonal 
polycrystal (Y-TZP), which has been widely used for prosthetic restorations. It will be shown that, as well as creep deformation, elastic recovery may also occur during holding period and affect significantly the determination of mechanical properties.

\section{Experimental}

Blocks of green stage Y-TZP (Doceram, Moeschter, Germany) were sintered at $1500{ }^{\circ} \mathrm{C}$ for $2 \mathrm{~h}$. The sintered samples were mounted in bakelite ground flat and then polished carefully with successively finer diamond paste to yield a scratch-free, mirror-like surface suitable for indentation.

The nanoindentation tests were carried out using a Berkovich diamond tip on a commercial nanoindenter (Nano Indenter G200, Agilent, Santa Clara, CA, USA). The indenter was loaded gradually to the prescribed peak load, $P_{\max }=400 \mathrm{mN}$, at a constant loading rate defined by $P_{\max } / t_{\mathrm{L}}$, where $t_{\mathrm{L}}$ is the prescribed loading time, and then was unloaded gradually at a constant unloading rate equal to the loading rate after being held at peak load for $120 \mathrm{~s}$.

For different tests, the loading time (unloading time) varied from 20 to $90 \mathrm{~s}$. This made the loading (unloading) rate examined in the present study varying in the range from $4.44(400 \mathrm{mN} / 90 \mathrm{~s})$ to $20(400 \mathrm{mN} / 20 \mathrm{~s}) \mathrm{mN} / \mathrm{s}$. For each prescribed $t_{\mathrm{L}}, 3-5$ indentations were made corresponding to each prescribed peak load level.

\section{Results and discussion}

Figure 1 shows the typical $P-h$ curves obtained in the present study. As can be seen, the $P-h$ curve measured with the lowest loading/unloading rate $\left(t_{\mathrm{L}}=90 \mathrm{~s}\right)$ is similar to those reported in the previous studies: during the entire test, the displacement increases with time. For tests with higher loading/unloading rates $\left(t_{\mathrm{L}}<90 \mathrm{~s}\right)$, however, an abnormal displacement change is observed during the holding period. In these cases, the displacements at the onset of the holding period, $h_{\mathrm{LM}}$, are observed to be larger than the displacement at the onset of the unloading period, $h_{\mathrm{UM}}$ (see the insert of Fig. 1). In other words, a significant displacement reduction occurs during the holding period. To our knowledge, the existence of a negative $\Delta h$ value has rarely been

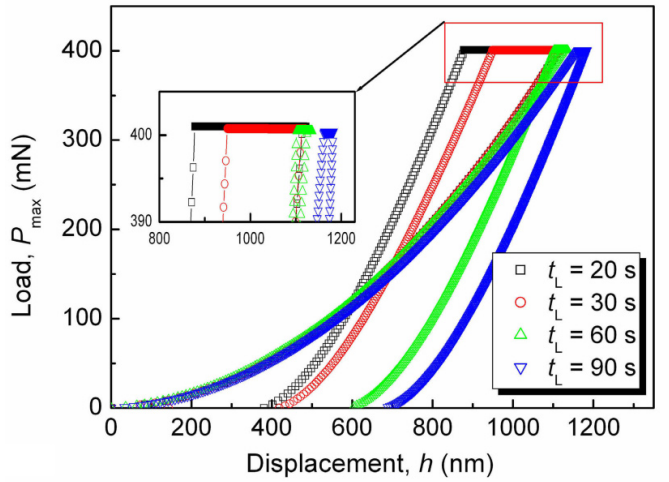

Fig. 1 Typical load-displacement $(P-h)$ curves measured in the present study for $P_{\max }=400 \mathrm{mN}$. The inset is the enlargement of the $P-h$ relation in the holding segment.

reported previously and warrants a further discussion.

Figure 2 shows the variation of the normalized displacement, $h / h_{\mathrm{LM}}$, with time during holding period measured with $P_{\max }=400 \mathrm{mN}$ and different loading time $t_{\mathrm{L}}$. It can be seen that, for $t_{\mathrm{L}}=90 \mathrm{~s}$, the normalized displacement increases monotonically with time, exhibiting a typical creep deformation. For $t_{\mathrm{L}}<90 \mathrm{~s}$, however, an increasing tendency in displacement is only observed at the initial stage of the holding period. With the prolonged time, the displacement decreases after reaching a maximum. This seems to indicate that, during the holding period, another unknown process, which may result in a decrease in displacement, would co-exist with creep because creep can only result in a continuous increase in displacement. Thus, the time dependence of the displacement, $h(t)$, during holding period may be written as

$$
h(t)=h_{0}+h_{\text {creep }}(t)+h_{\text {unknown }}(t)
$$

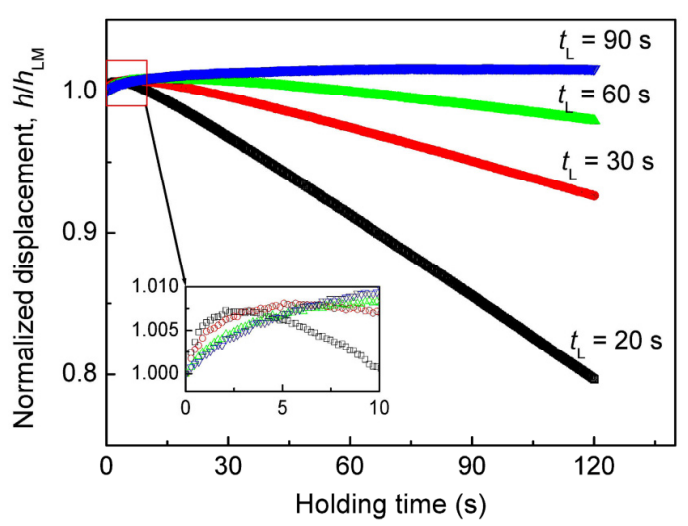

Fig. 2 Variation of the normalized displacement, $h / h_{\mathrm{LM}}$, with time during holding period measured with $P_{\max }=$ $400 \mathrm{mN}$ and different loading time. 
where $h_{\text {creep }}(t)$ and $h_{\text {unknown }}(t)$ are the displacements due to creep and the unknown process at time $t$, respectively, and $h_{0}$ is the displacement corresponding to the onset of the creep deformation.

As can be seen in the inset of Fig. 2, at the initial stage of the holding period, the $h(t)-t$ curves are very similar in shape with the typical nanoindentation creep curves observed in previous studies for metals [5], metallic glasses [10,11], coatings [12], and thin films [13]. The nanoindentation creep observed during the holding period was usually reported to be dependent strongly on the loading rate or strain rate, used during the loading period [10-13]. In general, the creep deformation increases with increasing loading rate. Similar tendency is also observed in the present study. As shown in the inset of Fig. 2, the indentation displacement rapidly increases with increasing loading rate or decreasing loading time, in the initial stage of the holding period. Based on these observations, one can infer that creep dominates the indentation deformation at the initial stage of the holding period.

For the curve measured with $t_{\mathrm{L}}=90 \mathrm{~s}$ (the lowest loading rate examined in the present study), the displacement tends to be constant when the holding period lasts for more than $80 \mathrm{~s}$, indicating that creep reaches the steady state, i.e., the stage with a constant creep rate. In this state, the displacement resulting from creep varies slightly and can be ignored. In other words, the above-mentioned unknown process which results in a decrease in displacement would dominate the indentation deformation at the subsequent stage of the holding period after the displacement reaches a maximum. Noting that, as the holding time $t$ prolongs, all the curves shown in the figure tend to be linear; to a first approximation, we may assume that the $h(t)-t$ relation associated with the unknown process, $h_{\text {unknown }}(t)$, may be treated as linear, i.e.,

$$
h_{\text {unknown }}(t)=A+B t
$$

where $A$ and $B$ are constants that can be derived directly by curve fitting.

Linear regression analyses were conducted for the experimental data shown in the holding time range from 80 to $120 \mathrm{~s}$ in Fig. 2 according to Eq. (2) to obtain the parameters $A$ and $B$ for each curve. And then, the displacement due to creep deformation can be deduced as

$$
h_{\text {creep }}(t)=h(t)-(A+B t)-h_{0}
$$

The results are now shown in Fig. 3. In constructing Fig. 3 , the parameter $h_{0}$ was set to let $h_{\text {creep }}(t)=0$ for $t=0$ in order to yield a curve starting from the origin of coordinates. As can be seen, all the curves shown in Fig. 3 behave very similar in both the shape and the loading rate dependence with the typical nanoindentation creep curve [10-13], giving an indirect support for the above conjecture that an unknown process, which may result in a decrease in displacement, would co-exist with creep during nanoindentation holding period. Figure 4 shows the best fit-value of the parameter $B$ in Eq. (2) as a function of loading time, $t_{\mathrm{L}}$. An evident loading rate dependence of the parameter $B$ is observed. Noting that $B$ has a negative value within the examined loading rate range, the decreasing tendency in $B$ implies that the effect of the unknown process on the displacement is more significant as the loading rate increases.

At present, we have not any direct evidences to analyze the origin of the above-mentioned unknown

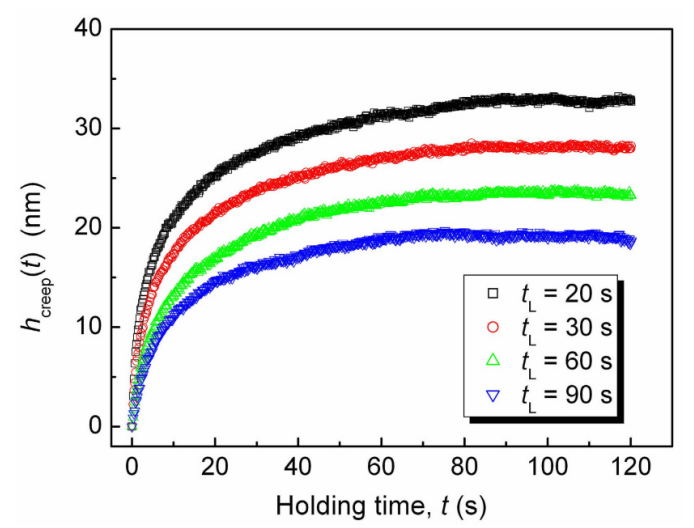

Fig. 3 Creep curves extracted by deducting the displacement resulting from the unknown process (Eq. (3)).

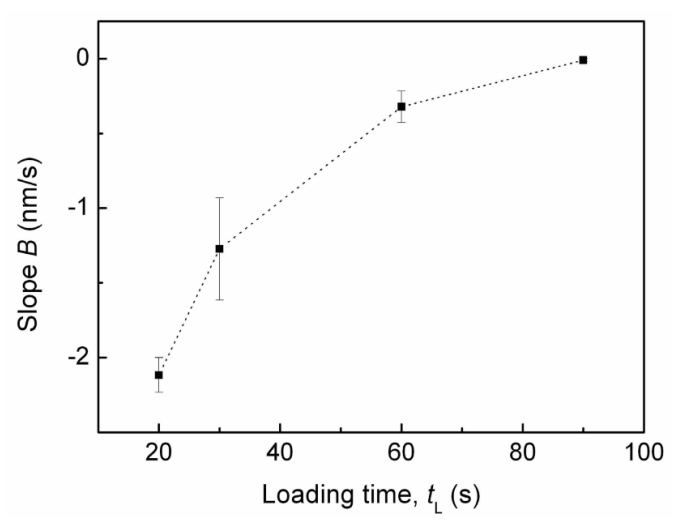

Fig. 4 Loading rate dependence of the parameter $B$ included in Eq. (2). 
process and its effect on the load-displacement relation. A possible explanation for the existence of such an unknown process may be the elastic recovery $[1,14,15]$, which is usually considered to occur only during the unloading period of an indentation test. In fact, there is reason to believe that elastic recovery may also occur during holding period as a result of stress relaxation due to creep. Furthermore, elastic deformation occurring during the loading period in indentation test is somewhat different with that in traditional bending or tension test. The loading rate for indentation test is generally larger and, in some cases, the loading period of an indentation test with a high loading rate may be approximately considered to be an impact procedure and results in an elastic deformation larger than that predicted with Hooke's law. This excess elastic deformation may recover during the holding period accompanied by the stress relaxation due to creep. Thus, a reduction in displacement may be expected. The loading rate dependence of the parameter $B$ shown in Fig. 4 confirms this analysis and indicates a larger displacement reduction would be expected to occur during holding period when a higher loading rate is used during loading period. Further experimental observations and analyses on this conjecture are now being conducted and will be reported in the near future.

The fact that the displacement reduction during holding period has rarely been reported in the previous studies may be explained as follows. First, most of the previous studies concern the metals or metallic glasses $[6,10,13]$ which exhibit significant plasticity during loading period and significant creep during holding period. The displacement change associated with the unknown process is comparatively small and has little effect on the displacement-time relation. Second, some studies were also made on brittle ceramics and glasses $[6,12,16]$, but with a small loading rate (low peak load to avoid the cracking due to brittleness) and/or a short holding time (considering the insignificant creep deformation). This may restrain the amount of the displacement reduction.

Now we turn to analyze the effect of the unknown process on the determination of hardness and Young's modulus.

In the present study, hardness, $H$, and Young's modulus, $E$, were calculated directly during each test by the data acquisition software of the nanoindenter based on the model of Oliver and Pharr [1], which automatically corrects the raw load-displacement data for the machine compliance and thermal drift. Here we quote directly the outputs of $H$ and $E$ for each individual unloading curve for discussion and the measured $H$ and $E$ are now plotted against the loading time in Fig. 5 (solid symbols), respectively. As can be seen, both $H$ and $E$ decrease with increasing loading time, i.e., decreasing loading rate. When the loading time is sufficiently long, both $H$ and $E$ tend to be invariable. Shao et al. [6] measured the hardness of the same Y-TZP with nanoindentation tests but with a loading time of $30 \mathrm{~s}$ and a holding time of $30 \mathrm{~s}$ and analyzed the experimental data with the modified proportional specimen resistance (PSR) model $[17,18]$. They reported that the material has a load-independent hardness of $18.0 \mathrm{GPa}$. In the present study, the hardness measured with a loading time of $90 \mathrm{~s}$ is $18.3 \pm 0.5 \mathrm{GPa}$, being in good agreement with the result of Shao et al. [6], implying a low loading (unloading) rate and a long holding time are the prerequisites for obtaining reliable hardness and Young's modulus, with nanoindentationtests.

The incredibly high values of the hardness and the Young's modulus obtained at high loading rate (short loading time) may be attributed to the displacement reduction associated with the above-mentioned unknown process. In the conventional OP method [1], the hardness, $H$, and the Young's modulus, $E$, are calculated with the following equations:

$$
\begin{gathered}
H=\frac{P_{\max }}{24.5 h_{\mathrm{c}}^{2}} \\
\frac{1}{E_{\mathrm{r}}}=\frac{1-v^{2}}{E}+\frac{1-v_{\mathrm{I}}^{2}}{E_{\mathrm{I}}} \\
E_{\mathrm{r}}=\left(\frac{\sqrt{\pi}}{2}\right) \frac{S}{\sqrt{24.5} h_{\mathrm{c}}}
\end{gathered}
$$

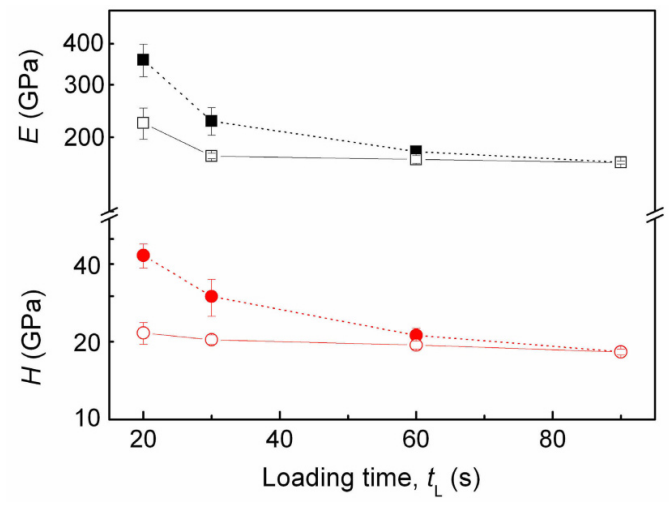

Fig. 5 Hardness and Young's modulus extracted as functions of loading time, $t_{\mathrm{L}}$. Solid symbols: uncorrected data quoted directly as the output of the data acquisition software; open symbols: data corrected with Eqs. (8) and (9). 
where $h_{\mathrm{c}}$ is the contact depth, $S$ is the contact stiffness, $E_{\mathrm{I}}$ and $v_{\mathrm{I}}$ are the Young's modulus and Poisson's ratio of the indenter respectively, and $v$ is the Poisson's ratio of the test material.

Based on Eqs. (4)-(6), the significant reduction in displacement during holding period would make the resultant $h_{\mathrm{c}}$ smaller than its true value, thereby enhancing the calculated results of the hardness and Young's modulus. Note that the parameter $B$ in Eq. (2) has a unit of $\mathrm{nm} / \mathrm{s}$ and therefore can be considered as the rate of displacement change associated with the unknown process during the holding period. In order to eliminate the effect of the displacement reduction, we may approximately correct the $h_{\mathrm{c}}$ determined with the conventional OP method by

$$
h_{\mathrm{c}}^{\text {corrected }}=h_{\mathrm{c}}+B t_{\mathrm{H}}
$$

where $t_{\mathrm{H}}$ is the total holding time and $t_{\mathrm{H}}=120 \mathrm{~s}$ in the present study. Thus, the hardness and the Young's modulus may be correspondingly corrected as

$$
\begin{gathered}
H=\frac{P_{\max }}{24.5\left(h_{\mathrm{c}}^{\text {corrected }}\right)^{2}} \\
E_{\mathrm{r}}=\left(\frac{\sqrt{\pi}}{2}\right) \frac{S}{\sqrt{24.5} h_{\mathrm{c}}^{\text {corrected }}}
\end{gathered}
$$

The corrected results are now also shown in Fig. 5 (open symbols). Clearly, such a correction restrains significantly the loading rate dependence of the extracted $H$ and $E$. In other words, reliable values of the hardness and the Young's modulus can be determined within a wide range of loading rate.

\section{Conclusions}

An abnormal displacement change observed during the holding period in nanoindentation tests on an yttria-stabilized tetragonal zirconia polycrystal (Y-TZP) was reported in this paper. When conducting nanoindentation tests on Y-TZP with a high loading rate (short loading time), the indenter displacement exhibits a significant reduction during the holding period after experiencing a short increase due to creep. The degree of displacement reduction during holding period decreases with decreasing loading rate, and when the loading rate descresaes to be smaller than about 4.44 $\mathrm{mN} / \mathrm{s}$, the displacement-time curve behaves the same as the typical nanoindentation creep curves reported in previous studies. This experimental phenomenon has rarely been reported previously and strongly implies that, during the holding period, another unknown process, which may result in a decrease in displacement, would co-exist with creep because creep can only result in a continuous increase in displacement. Elastic recovery was suggested to be one of the possible sources for such an unknown process.

An empirical approach was proposed in this study to describe the displacement-time relation associated with the unknown process. It was shown that, with this empirical approach, reliable nanoindentation creep curve, as well as the comparable hardness and Young's modulus, may be extracted from data analyses.

To our knowledge, this is the first time to observe such an abnormal phenomenon and the universality and origin of this abnormal phenomenon should be examined carefully and critically in the future. The analyses and the approach presented here may be helpful for the further studies on the same or similar phenomena.

\section{Acknowledgements}

This work was supported by the Opening Project of State Key Laboratory of High Performance Ceramics and Superfine Microstructure, China.

\section{References}

[1] Oliver WC, Pharr GM. An improved technique for determining hardness and elastic modulus using load and displacement sensing indentation experiments. J Mater Res 1992, 7: 1564-1583.

[2] Zeng K, Söderlund E, Giannakopoulos AE, et al. Controlled indentation: A general approach to determine mechanical properties of brittle materials. Acta Mater 1996, 44: $1127-1141$.

[3] Malzbender J, de With G, den Toonder J. The $P-h^{2}$ relationship in indentation. $J$ Mater Res 2000, 15: 1209-1212.

[4] Fischer-Cripps AC. A simple phenomenological approach to nanoindentation creep. Mat Sci Eng A 2004, 385: 74-82.

[5] Goodall R, Clyne TW. A critical appraisal of the extraction of creep parameters from nanoindentation data obtained at room temperature. Acta Mater 2006, 54: 5489-5499.

[6] Shao L, Jiang D, Gong J. Nanoindentation characterization of the hardness of zirconia dental ceramics. Adv Eng Mater 2013, 15: 704-707.

[7] Zhang Y, Allahkarami M, Hanan JC. Measuring residual stress in ceramic zirconia-porcelain dental crowns by nanoindentation. J Mech Behav Biomed 2012, 6: 120-127.

[8] Meng Z, Jiang D. Measuring mechanical properties of zirconia dental crowns by nanoindentation. Key Eng Mater 
2014, 591: 150-153.

[9] Alao A-R, Yin L. Nanoindentation characterization of elasticity, plasticity and machinability of zirconia. Mat Sci Eng A 2015, 628: 181-187.

[10] Wang F, Li JM, Huang P, et al. Nanoscale creep deformation in Zr-based metallic glass. Intermetallics 2013, 38: $156-160$.

[11] Chen YH, Huang JC, Wang L, et al. Effect of residual stresses on nanoindentation creep behavior of Zr-based bulk metallic glasses. Intermetallics 2013, 41: 58-62.

[12] Guo S, Kagawa Y. Effect of loading rate and holding time on hardness and Young's modulus of EB-PVD thermal barrier coating. Surf Coat Technol 2004, 182: 92-100.

[13] Ma Z, Long S, Pan Y, et al. Loading rate sensitivity of nanoindentation creep in polycrystalline Ni films. J Mater Sci 2008, 43: 5952-5955.

[14] Sneddon IN. The relation between load and penetration in the axisymmetric Boussinesq problem for a punch of arbitrary profile. Int J Eng Sci 1965, 3: 47-57.

[15] Lawn BR, Howes VR. Elastic recovery at hardness indentations. J Mater Sci 1981, 16: 2745-2752.

[16] Chakraborty R, Dey A, Mukhopadhyay AK. Loading rate effect on nanohardness of soda-lime-silica glass. Metall Mater Trans A 2010, 41: 1301-1312.

[17] Gong J, Wu J, Guan Z. Examination of the indentation size effect in low-load Vickers hardness testing of ceramics. J Eur Ceram Soc 1999, 19: 2625-2631.

[18] Jiang D. Recent progress in the phenomenological description for the indentation size effect in microhardness testing of brittle ceramics. $J$ Adv Ceram 2012, 1: 38-49.

Open Access The articles published in this journal are distributed under the terms of the Creative Commons Attribution 4.0 International License (http://creativecommons.org/licenses/ by/4.0/), which permits unrestricted use, distribution, and reproduction in any medium, provided you give appropriate credit to the original author(s) and the source, provide a link to the Creative Commons license, and indicate if changes were made. 